\title{
EDITORIAL
}

\section{DNA and Huntington's chorea ${ }^{1}$}

Huntington's chorea (HD) is a genetic disease transmitted as an autosomal dominant. The prevalence is 7 per 100000 , and about 3500 people in Britain are affected (Caro, 1977; Walker et al. 1981). The disease affects primarily the basal ganglia and manifests itself as chorea, a characteristic type of progressive dementia (Folstein et al. 1983), and frequently other psychiatric syndromes (Dewhurst, 1970; Caine \& Shoulson, 1983). Only palliative treatment is available, and the disease progresses through severe disability to death in an average of 15 years. HD most frequently begins in middle age when reproduction has taken place. Offspring are at $50 \%$ risk of developing the disease and there are probably about 20 per 100000 asymptomatic gene carriers in Britain (Harper et al. 1979) or about 100000 in the UK. There is, at present, no practical means of detecting unaffected gene carriers.

\section{GENETIC COUNSELLING}

At present, the only way of limiting the disease is by avoiding conception or terminating pregnancy. A programme putting this into effect has been started in a well demarcated population in Wales (Harper et al. 1979) where, on the basis that the risk is $50 \%$ for offspring and sibs of an affected person, $25 \%$ for second-degree and $12.5 \%$ for third-degree relatives, genetic counselling is offered within a supportive framework. Young relatives of affected persons are given advice on the disease, together with information on contraception, termination and adoption. An assessment after 9 years gave some indication that the number of at-risk births had been reduced by this measure.

\section{GENETIC LINKAGE}

A further approach has been the attempt to detect asymptomatic carriers by using neighbouring genes. A gene is transmitted as part of a segment of DNA which is inherited as a coherent block from a parent. Consequently, neighbouring genes will usually be inherited together, since they are physically attached (linked) as part of the same chromosome. The closer they are together, the less likely are they to become separated at meiosis, when every chromosome pairs with its partner and, as a result of various cut-and-join mechanisms, each chromosome transmitted is usually compounded of two or three segments from the paired parental chromosomes.

For example, if the parental chromosome pair is

$$
\text { ABCDEFGHIJKLM and abcdefghijklm }
$$

then the chromosome passed on might be

$$
\text { ABCDefghijKLM abcDEFGHIJKLM or ABcdefgHIJKLM. }
$$

The chromosome passed on is sometimes known as a haplotype. In the example above, if the letters referred to genes, and if in the individual concerned we could distinguish $G$ and $g$ by some test, but not $\mathrm{H}$ and $\mathrm{h}$, then we could predict the presence of $\mathrm{H}$ from $\mathrm{G}$. In the third case, however, which represents a recombination, $G$ and $H$ have separated and the prediction would be wrong.

If we relate this to the practical problem of Huntington's chorea, and continue with the $G, g$ symbolism for the gene, we can detect and distinguish (the marker) and use $\mathrm{H}, \mathrm{h}$ for the Huntington locus ( $\mathrm{H}$ indicating the Huntington gene and $\mathrm{h}$ the normal gene). Various possibilities then arise. If the loci $G$ and $H$ are so close that they rarely recombine and the only mutation from $h$ to $H$

\footnotetext{
1 Address for correspondence: Dr D. C. Watt, St John's Hospital, Stone, Aylesbury, Bucks HP17 8PP.
} 
occurred in a chromosome with the G form, then only the pairs gh, Gh and GH would be found in the population and anyone without a $\mathrm{G}$ gene could not inherit this mutation (i.e. $\mathrm{H}$ ). In practice, however, there are four difficulties which obstruct a prediction made on this basis.

(1) The possibility of recombination cannot be eliminated. Even if it only occurs once in, say, twenty meioses, the events underlying gamete formation, it would then have a frequency of 1 in 20 . In this case an inference relating two cousins, one affected, could be in error by $4 / 20$, as they are separated by four meiotic events.

(2) The affected parent may not be informative due to the two genes at the $G$ locus being the same, or due to one being identical with one present in the other parent. This difficulty should be reduced as DNA techniques come into use, with the recognition of variation at several nearby segments of DNA. It is difficult to produce a general description of informative and uninformative families, but not usually difficult to see the potential by studying the pedigree of a known family.

(3) The requisite information may not be available for the family concerned. In order for a potential carrier to be diagnosed, before or after birth, it is necessary that the phase of the pair of loci is known in the family, i.e. does $\mathrm{H}$ go on the same chromosome with $\mathrm{G}$ or $\mathrm{g}$ ? This will usually require DNA from one affected individual and from at least one parent of the individual at risk. Occasionally, the phase may be inferred from a surviving spouse of the affected individual. For example, if parents $\mathrm{H}$ ?/h? and hg/hg have an affected child who has the genes $\mathrm{G}$ and $\mathrm{g}$, and is therefore of haplotype $\mathrm{Hg} / \mathrm{hG}$ or $\mathrm{HG} / \mathrm{hg}$, then, since one parent could only have provided the haplotype hg, the child must be $\mathrm{HG} / \mathrm{hg}$. Clearly, as the manifestation of the disease is not usually unambiguous before the late thirties, such inferences can only benefit a minority of potential parents until there are facilities for the storage of DNA.

(4) The diagnosis may be wrong. A linked gene can then provide only erroneous prognoses. However, the preservation of DNA may eventually allow the refutation of this diagnosis. Unless all cases were due to a unique mutation (i.e. all cases arose from an error at the same single locus) this would be extremely difficult and might be impossible. For example, the exclusion of a diagnosis of thalassaemia of unknown type would not be possible without assuming a limited range of possibilities and excluding all forms not yet discovered (Weatherall, 1982). Various authors have produced evidence for the possibility that HD is genetically heterogeneous: Caro (1977), for instance, has collected data suggesting that ages of death of HD subjects correlate in families and are distributed bimodally. Wallace \& Hall (1972) and Went et al. (1975) have shown greater intrafamilial than interfamilial similarity in age of onset and age at death data.

The most widely used markers are characteristics of blood such as blood groups. In a major collaborative search for linkage, results from Welsh, English, Dutch, North American and Australian Huntington's families were combined to give more than 121 families and 1718 examined individuals, affected and unaffected (Volkers et al. 1980). Thirty-two markers were used and no evidence for linkage was found. A useful outcome of this study, however, was that the locus for Huntington's Chorea could be excluded from about one-fifth of the total human genome. It is clear that identifying asymptomatic carriers by genetic linkage studies is likely to be a long haul.

\section{MOLECULAR GENETICS}

The new DNA techniques allow the detection of variability in any neighbouring loci, although there are formidable difficulties in finding a neighbourhood sufficiently close to a particular locus. Since DNA is distributed throughout all tissues, the genetic lesion can, in principle, be defined from any tissue, including blood, even if the disease is expressed only in some inaccessible site such as the brain, eye or ear. The recent discovery of a nearby probe increases the precision of a genetic prognosis, opens the way to the identification of the underlying lesion, and clarifies whether mutation is sufficiently rare to be ignored in practice.

The molecular structure of DNA resembles a ladder twisted on its longitudinal axis, in which the rungs represent the genetically active component. Each rung is formed from pairs of bases which are of two types; either adenine with thymine or guanine with cytosine. Each gene is a specific 
sequence of these bases which acts as a code determining the constituent amino acids from which enzymes and other proteins are formed. DNA techniques enable these base pairs to be divided, thus separating the two strands of DNA on which the bases are strung. Such separated strands will recombine, but only where the sequence of bases on each correspond exactly over at least twenty or so pairs. Through a modified intermediary (messenger RNA), which copies a mirror image of the base sequence code, DNA determines the particular order of amino acids used in building up a specific protein, triplets of bases being sufficient to specify each amino acid. A synthesized copy of a DNA sequence (complementary or cDNA) can be made which will lock on specifically to a corresponding sequence of DNA. The fragment of synthetic DNA can be made radioactive and used as a probe to seek a corresponding portion of DNA, thus marking it and making it detectable. By the use of certain enzymes (restriction endonucleases) a chromosome, which contains about 100 million bases, can be sectioned longitudinally at specific locations into varied lengths of DNA usually averaging a few thousand bases long. These can be separated out according to length by electrophoresis and marked with probes, thus giving a characteristic individual pattern in relation to any probe.

\section{GENE MAPPING}

In order to use these features of DNA in locating the HD gene, therefore, a search must be made for a probe which will lock on only to fragments of DNA near to the locus. This would be revealed by association of the probe with the DNA of affected subjects who have an identical mutation. The probe must then be tried on a larger number of families to confirm the result and estimate the frequency of recombinations. This indicates the frequency of diagnostic error to be expected, and if it is unacceptable a probe nearer to the locus must be found. Where probes are available for the gene itself variants within, or very close to the gene, can be found, as in the haemoglobinopathies (Weatherall, 1982). This stage has been reached in some disorders, including phenylketonuria, Christmas disease and Lesch-Nyhan syndrome.

A probe (labelled G8) has now been found which is likely to be within a tenth of a chromosome of the HD locus on chromosome 4 (Gusella et al. 1983). This work was based on two large families in North and South America, in each of which the allele defined by G8 and associated with HD was different. This result is now being confirmed using other families.

\section{NEW MUTATIONS}

The frequency of new mutations giving rise to HD will assume new significance once a satisfactory method of diagnosis is in use. Obviously, the new method will be powerless to detect the aberrant gene before HD manifests itself; nor will it be able to define its phase until a second case has occurred. The gene will then already have been passed on. The absence of family history in affected subjects is the main method of detection of new mutants at present and, as Shaw \& Caro (1982) have shown, it is arduous, exacting and fallible. Investigation by the use of rare DNA variants close to the gene offers the prospect of a more reliable method of estimation since these, if they appear to follow the Huntington gene, are unlikely to be due to crossing over in ancestral lines. If, for example, two apparently unrelated cases had the same rare allele at a neighbouring locus they would probably be descended from the same affected ancestor.

\section{CLINICAL APPLICATION}

The DNA of unaffected potential gene carriers could be scanned by a probe, once one is found reliably linked to the HD locus and the phase is known. In addition, it could then be used for antenatal diagnosis. This has been possible hitherto, where a probe is available, by culture of foetal cells from amniotic fluid and extraction of DNA. Direct preparations are difficult, as much degraded DNA is present. However, this process takes several weeks and, if protracted studies are needed, 
the results can be too late to permit termination of pregnancy in the event of a positive result. An alternative method of obtaining foetal DNA now being developed is chorion biopsy. This is carried out at 8 weeks of pregnancy; it provides good quality DNA directly and promises to be a more useful method.

The development of a satisfactory probe, with the prospect of reliable identification of gene carriers in the first few months of foetal life, or postnatally before the reproductive period, now seems within sight. Once it has been established, the grave consequences of misdiagnosis will loom larger than they do now, affecting mainly descendants but also siblings of probands. The diagnosis of subjects and their relation to the proband is therefore crucial. In five years we shall be reliant solely on the case records of some HD subjects now alive and it is therefore imperative that the grounds on which a diagnosis has been made are fully and clearly documented, including the clinical findings, post-mortem reports and family histories. The last are particularly prone to fallibility. It has been recorded, for instance, that $50 \%$ of referrals investigated at a specialist hospital are without a family history (Heathfield, 1968). This is so far at variance with other thorough investigations of HD families that it indicates either a grossly unrepresentative sample or insufficient family investigation, or more probably both. It is still more important that blood from affected or elderly subjects from HD families, and where possible their spouses, is now stored so that the diagnosis can be confirmed from the DNA when a probe is satisfactorily developed and a family blood relationship can be confirmed through blood groups.

Fortunately, DNA is fairly stable in blood, normally surviving without degradation sufficiently long to withstand postage, after which it is stable for up to a week at $4{ }^{\circ} \mathrm{C}$, and for months if frozen at $-40^{\circ} \mathrm{C}$ or preferably $-70^{\circ} \mathrm{C}$. It is virtually indefinitely stable after extraction. It should be taken in ethylene diamine tetra acetic acid (EDTA). Each test involves the DNA from less than $0.5 \mathrm{ml}$; $20 \mathrm{ml}$, preferably with duplication of storage, should be sufficient.

The elucidation of the structure and mechanism of the HD gene, with prospects of ameliorative treatment, is a future possibility which will require the resources, experience and long-term effort of a department undertaking large-scale DNA research. Recent advances in DNA technology have greatly improved the prospect for $\mathrm{HD}$, but its realization demands preparation now if the advantage is to be secured for future generations potentially at risk.

D. C. WATT AND JOHN EDWARDS

\section{REFERENCES}

Caine, E. D. \& Shoulson, I. (1983). Psychiatric syndromes in Huntington's Disease. American Journal of Psychiatry 140, 728-733.

Caro, A. J. (1977). A genetic problem in East Anglia: Huntington's Chorea. Thesis for Ph.D: University of East Anglia.

Dewhurst, K. (1970). Personality disorder in Huntington's Disease. Psychiatrica Clinica 3, 221-229.

Folstein, S. E., Abbott, M. H., Chase, G. A., Jensen, B. A. \& Folstein, M. F. (1983). The association of affective disorders with Huntington's Disease in a case series and in families. Psychological Medicine 13, 537-542.

Gusella, J. F., Wexler, N. S., Conneally, P. M., Naylor, S. L., Anderson, M. A., Tanzi, R. E., Watkins, P. C., Ottina, K. Wallace, M. R., Sakaguchi, A. Y., Young, A. B., Shoulson, I. Bonilla, E. \& Martin, J. B. (1983). A polymorphic DNA marker genetically linked to Huntington's Disease. Nature 306, 234-238.

Harper, P. S., Walker, D. A., Tyler, A., Newcombe, R. G. \& Davies, K. (1979). Huntington's Chorea - the basis for long-term prevention. Lancet ii, 346-349.
Heathfield, K. W. G. (1968). Huntington's Chorea : investigation into the prevalence of this disease in the area covered by the N.E. Metropolitan Regional Board. Brain 90, 203-232.

Shaw, M. \& Caro, A. (1982). The mutation rate to Huntington's Chorea. Journal of Medical Genetics 19, 161-167.

Volkers, W. S., Went, L. N., Vegter-Van der Vlis, M., Harper, P. S. \& Caro, A. (1980). Genetic linkage studies in Huntington's Chorea. Annals of Human Generics 44, 75-79.

Walker, D. A., Harper, P. S., Wells, C. E. C., Tyler, A., Davies, K. \& Newcombe, R. G. (1981). Huntington's Chorea in South Wales. Clinical Genetics 19, 213-221.

Wallace, D. C. \& Hall, A. C. (1972). Evidence of genetic heterogeneity in Huntington's Chorea. Journal of Neurology, Neurosurgery and Psychiatry 33, 789-800.

Weatherall, D. J. (1982). The New Genetics and Clinical Practice. Nuffield Provincial Hospitals Trust: London.

Went, L. N., Vegter-Van der Vlis, M., Volkers, W. \& Collewijn, H. (1975). Huntington's Chorea. In Early Diagnosis and Prevention of Inherited Disease (ed. L. N. Went, C. Verney-Keers and A. G. J. M. Van der Linden), pp. 13-25. Leiden University Press. 